\title{
Respostas fisiológicas do girassol em fase reprodutiva ao estresse hídrico e salino
}

\author{
Physiological response of sunflower in the reproductive phase to water and salt stress \\ Respuestas fisiológicas del girasol en fase reproductiva al estrés hídrico y salino
}

Recebido: 03/09/2021 | Revisado: 12/09/2021 | Aceito: 13/09/2021 | Publicado: 14/09/2021

Alisson Macendo Amaral
ORCID: https://orcid.org/0000-0003-3035-2763
Instituto Federal de Educação, Ciência e Tecnologia do Norte de Minas Gerais, Brasil
E-mail: alisson.amaral@ifnmg.edu.br
Alefe Viana Souza Bastos
ORCID: https://orcid.org/0000-0002-0349-2421
E-mail: alefe_viana@ @otmail.com
Instituto Federal de Educação, Ciência e Tecologia Goiano, Brasil
Maria Ângela Cruz Macêdo dos Santos
ORCID: https://orcid.org/0000-0003-1418-7438
Universidade Federal do Recôncavo da Bahia, Brasil
E-mail: angelacruzmacedo@gmail.com
Marconi Batista Teixeira
ORCID: https://orcid.org/0000-0002-0152-256X
E-mail: marconi.teixeira@ @ifgoiano.edu.br
Frederico Antonio Loureiro Soares
ORCID: https://orcid.org/0000-0002-4152-5087
Instituto Federal de Educacão, Ciência e Tecnologia Goiano, Brasil
E-mail: frederico.soares@ifgoiano.edu.br

\section{Resumo}

O objetivo do estudo foi compreender as alterações fisiológicas do girassol, em fase reprodutiva, quando submetido a estresses hídrico e salino. $\mathrm{O}$ estudo foi conduzido em ambiente protegido, aplicando-se 4 níveis de irrigação (25\%, $50 \%, 75 \%$ e $100 \%$ da evapotranspiração da cultura) e 2 níveis de condutividade elétrica da água de irrigação (0,6 e 3,0

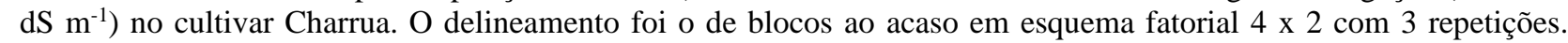
Foram avaliados parâmetros de trocas gasosas e fluorescência. Para as trocas gasosas avaliou-se a taxa fotossintética (A), taxa transpiratória $(\mathrm{E})$, condutância estomática $(\mathrm{Gs})$, concentração interna de $\mathrm{CO}_{2}(\mathrm{Ci})$ e as razões $\mathrm{Ci} / \mathrm{Ca}, \mathrm{A} / \mathrm{Ci}$, A/Gs e A/E. Para a fluorescência da clorofila A foram mensurados o rendimento quântico efetivo do fotossistema II ( $\phi$ PSII), o rendimento quântico potencial do fotossistema II (Fv/Fm) o quenching fotoquímico (qP) e o quenching não-fotoquímico (qNP). A fluorescência da clorofila A não sofreu alterações significativas com os tratamentos. A Charrua demonstrou adaptabilidade aos efeitos dos estresses, resultando em diminuição da abertura estomática, aumento de Gs, da A, da eficiência instantânea de carboxilação e eficiência do uso da água quando submetido aos menores níveis de irrigação e condutividade elétrica.

Palavras-chave: Helianthus annus L.; Adaptabilidade; Resistência.

\begin{abstract}
The objective of the study was to understand the physiological changes of the sunflower, in the reproductive phase, when submitted to water and saline stresses. The study was conducted in a protected environment, applying 4 irrigation levels $(25,50,75$ and $100 \%$ of crop evapotranspiration) and 2 levels of electrical conductivity of irrigation water $\left(0.6\right.$ and $\left.3.0 \mathrm{dS} \mathrm{m}^{-1}\right)$ in cultivar Charrua. The design was a randomized complete block design in a $4 \times 2$ factorial scheme with 3 replicates. Parameters of gas exchange and fluorescence were evaluated. For the gas exchange the photosynthetic rate (A), transpiratory rate $(\mathrm{E})$, stomatal conductance $(\mathrm{Gs})$, internal $\mathrm{CO}_{2}$ concentration $(\mathrm{Ci})$ and the $\mathrm{Ci} / \mathrm{Ca}, \mathrm{A} / \mathrm{Ci}, \mathrm{A} / \mathrm{Gs}$ and $\mathrm{A} / \mathrm{E}$. For the chlorophyll A fluorescence, the effective quantum yield of photosystem II $(\varphi$ PSII), potential quantum yield of photosystem II ( $\mathrm{Fv} / \mathrm{Fm})$ and photochemical quenching $(\mathrm{qP})$ and non-photochemical quenching (qNP) were measured. The chlorophyll A fluorescence did not change significantly with the treatments. The Charrua showed adaptability to the effects of the stresses, resulting in a decrease in stomatal opening, increases of Gs, A, instantaneous carboxylation efficiency and water use efficiency when submitted to lower levels of irrigation and electrical conductivity.
\end{abstract}

Keywords: Helianthus annus L.; Adaptability; Resistance. 


\begin{abstract}
Resumen
El objetivo del estudio fue comprender los cambios fisiológicos del girasol, en la fase reproductiva, cuando se somete a estrés hídrico y salino. El estudio se realizó en un ambiente protegido, aplicando 4 niveles de riego $(25 \%, 50 \%, 75 \%$ y $100 \%$ de evapotranspiración del cultivo) y 2 niveles de conductividad eléctrica del agua de riego $\left(0,6\right.$ y $\left.3,0 \mathrm{dS} \mathrm{m} \mathrm{m}^{-1}\right)$ en cultivar Charrua. El diseño fue de bloques al azar en un esquema factorial 4 x 2 con 3 repeticiones. Se evaluaron los parámetros de intercambio de gases y fluorescencia. Para el intercambio de gases, la tasa fotosintética (A), la tasa de transpiración (E), la conductancia estomática (Gs), la concentración interna de $\mathrm{CO} 2(\mathrm{Ci})$ y las relaciones $\mathrm{Ci} / \mathrm{Ca}$, $\mathrm{A} / \mathrm{Ci}, \mathrm{A} / \mathrm{Gs}$ y A/E. Para la fluorescencia de la clorofila A, se midió el rendimiento cuántico efectivo del fotosistema II ( $\phi$ PSII), el rendimiento cuántico potencial del fotosistema II (Fv / Fm), la extinción fotoquímica (qP) y la extinción no fotoquímica (qNP). La fluorescencia de la clorofila A no cambió significativamente con los tratamientos. El Charrua mostró adaptabilidad a los efectos de las tensiones, lo que resultó en una disminución en la apertura estomática, un aumento en Gs, A, eficiencia de carboxilación instantánea y eficiencia en el uso del agua cuando se sometió a niveles más bajos de riego y conductividad eléctrica.
\end{abstract}

Palabras clave: Helianthus annus L.; Adaptabilidad; Resistencia.

\title{
1. Introdução
}

A irrigação é o método de reposição de água no solo que visa suprir as necessidades hídricas dos cultivos. Entretanto, as reservas de água doce disponíveis a este fim estão cada vez mais escassas, principalmente pelas altas taxas de evapotranspiração aliadas a regimes pluviométricos insuficientes.

A expansão populacional torna a irrigação uma ferramenta indispensável ao aumento da produção agrícola (Feng et al., 2017). Deve-se considerar uma alternativa viável à gestão dos recursos hídricos o uso de água de qualidade inferior, como aquelas de elevada condutividade elétrica, aliadas a irrigações deficitárias, que impõe um certo nível de estresse à planta durante um período específico ou ao longo do ciclo (Lira et al., 2015; Gomes et al., 2015; Patane et al., 2017).

Considerando o estresse imposto a um cultivo de interesse econômico, devido à aplicação de água de qualidade inferior, a produtividade satisfatória só será obtida se a cultura for resistente e, nesse sentido, o girassol (Helianthus annus L.) possui destaque.

O girassol é uma oleaginosa de grande interesse econômico devido as suas características de adaptabilidade a condições edafoclimatológicas diversas e agronômicas (Lustri et al., 2017; Dalchiavon et al., 2016), como a produção de óleo (Guedes Filho et al., 2015) e de flores (Santos Jr. et al., 2016), por exemplo. Atualmente é considerada uma das mais importantes culturas de sementes oleaginosas com tolerância moderada a alta à seca e diferentes níveis de salinidade (Mila et al., 2017),

A salinidade em níveis elevados influencia mais negativamente a fotossíntese e o crescimento celular, a absorção e distribuição de água e nutrientes, além do risco constante da vulnerabilidade à formação das espécies reativas de oxigênio, ocasionando danos oxidativos nas células (Habibi, 2017; Ashraf et al., 2017).

A maioria das culturas possui períodos críticos quando submetidas a situações de deficiência hídrica, durante os quais a falta de água pode levar a alterações no desenvolvimento das plantas e consequente redução da produtividade final (Miorini et al., 2011). No girassol, a fase mais crítica ao déficit hídrico é o período compreendido entre 10 a 15 dias antes do início do florescimento e 10 a 15 dias após o final da floração (Dutra et al., 2012).

As respostas fisiológicas do cultivo do girassol sobre deficiência hídrica e salina ainda são incipientes e pouco conclusivas. As respostas de uma planta a estresses dependem da espécie, do genótipo, do estádio fenológico e do período de exposição às condições salinas (Santos \& Brito, 2016).

O entendimento do comportamento fisiológico do girassol, em principal na fase reprodutiva, é de fundamental importância para entender como estresses abióticos impostos ao cultivo influenciam processos posteriores, como crescimento e produtividade. Diante disso, objetivou-se compreender o comportamento fisiológico do girassol em estádio reprodutivo, quando submetido a estresses hídrico e salino, por meio de avaliações de trocas gasosas e fluorescência da clorofila. 


\section{Metodologia}

$\mathrm{O}$ experimento foi conduzido em ambiente de cultivo protegido não climatizado, localizado em área experimental do Instituto Federal Goiano - Campus Rio Verde, com coordenadas correspondentes a $17^{\circ}$ 48' 3,52"S de latitude, 50 54' 27,33"O de longitude e altitude média de $720 \mathrm{~m}$. O ambiente de cultivo possui dimensões correspondentes a 17,6 m de comprimento, 7 $\mathrm{m}$ de largura e 6,0 $\mathrm{m}$ de altura, revestida lateralmente por tela com fator de sombreamento igual a $11,5 \%$ e revestimento do teto com lona plástica transparente de 150 micras.

O delineamento utilizado foi o de blocos casualizados em esquema fatorial 4 x 2 com 3 repetições, cujos tratamentos foram 4 níveis de irrigação (25, 50, 75 e 100\% da evapotranspiração de referência) e 2 níveis de condutividade elétrica da água de irrigação $\left(0,6\right.$ e 3,0 dS m $\left.\mathrm{m}^{-1}\right)$. O nível de condutividade elétrica da água de irrigação (CE) igual a $0,6 \mathrm{dS} \mathrm{m}^{-1}$ correspondia a salinidade natural da água usada nas irrigações e mensurada na fonte de abastecimento, enquanto que a maior CE $\left(3,0 \mathrm{dS} \mathrm{m}^{-1}\right)$ foi escolhida por apresentar o máximo do intervalo usual para avaliar a qualidade da água de irrigação pelo critério da salinidade potencial (Almeida, 2010). Segundo este autor, as condutividades elétricas da água de irrigação do estudo em questão, correspondem a nenhuma restrição de uso $\left(<0,7 \mathrm{dS} \mathrm{m}^{-1}\right)$ e ligeira a moderada restrição de uso $\left(0,7\right.$ a $\left.3 \mathrm{dS} \mathrm{m}^{-1}\right)$ da água na irrigação.

As unidades experimentais constaram de vasos com volume de $60 \mathrm{~L}$, cheios por um Latossolo Vermelho Distroférrico de textura média, cujas características e propriedades físico-hídricas são informadas na Tabela 1. Cada vaso apresenta em sua parte interior uma camada drenante composta por $1 \mathrm{~cm}$ de areia lavada, manta do tipo Bidin e brita $\mathrm{n}^{\circ} 1$. A adubação foi calculada conforme Novais et al. (1991) para ensaios em vaso.

Tabela 1. Propriedades físico-hídricas do solo usado no experimento.

\begin{tabular}{|c|c|c|c|c|c|c|c|}
\hline Profundidade & Ds & Dp & PT & Macro & Micro & $\mathrm{CV}$ & PMP \\
\hline$(\mathrm{cm})$ & \multicolumn{2}{|c|}{$\left(\mathrm{g} \mathrm{cm}^{-3}\right)$} & \multicolumn{3}{|c|}{$(\%)$} & \multicolumn{2}{|c|}{$\left(\mathrm{m}^{3} \mathrm{~m}^{-3}\right)$} \\
\hline $0-20$ & 1,02 & 2,68 & 0,62 & 0,12 & 0,50 & 51,83 & 30,50 \\
\hline $20-40$ & 0,97 & 2,80 & 0,65 & 0,10 & 0,55 & 55,00 & 31,33 \\
\hline Profundidade & & Textura & & \multicolumn{4}{|c|}{ Velocidade de infiltração básica } \\
\hline \multirow[t]{2}{*}{$(\mathrm{cm})$} & \multicolumn{3}{|c|}{$\left(\mathrm{g} \mathrm{kg}^{-1}\right)$} & \multicolumn{4}{|c|}{$\left(\mathrm{mm} \mathrm{h}^{-1}\right)$} \\
\hline & Areia & Silte & Argila & \multirow{2}{*}{\multicolumn{4}{|c|}{26,84}} \\
\hline $0-20$ & 458,3 & 150,2 & 391,5 & & & & \\
\hline $20-40$ & 374,9 & 158,3 & 466,8 & & & & \\
\hline
\end{tabular}

Ds - Densidade global; Dp - Densidade de partículas; PT- Porosidade total; Macro- Macroporosidade; Micro - Microporosidade; CV Capacidade de vaso e PMP - Ponto de murcha permanente.

Fonte: Autores (2021).

Os níveis de irrigação (NI) foram manejados através do balanço de água do solo no interior de um lisímetro de pesagem por células de carga, com dimensões representativas às unidades experimentais. As irrigações foram realizadas diariamente visando repor a umidade do solo perdida por evapotranspiração e proporcionais as frações usadas nos tratamentos (25, 50, 75 e 100\% da Eto). Inicialmente o lisímetro teve sua massa elevada até umidade da capacidade de campo e nas irrigações sucessivas, quantificou-se a variação da massa perdida por evapotranspiração para que fosse reposta via sistema de irrigação.

A CE de 3,0 dS m ${ }^{-1}$ foi obtida com adição de NaCl, P.A - ACS (CAS - 7647-14-5) utilizando-se a Eq.1 proposta por Richards (1954). 
$Q_{\text {NaCl }}=640\left(C E_{a d}-C E_{a i}\right)$

em que:

$\mathrm{Q}_{\mathrm{NaCl}}$ - quantidade de $\mathrm{NaCl}$ exigida para elevar a $\mathrm{CE}$ até a desejada, em $\mathrm{mg} \mathrm{L}^{-1}$;

$\mathrm{CE}_{\mathrm{ad}}$ - condutividade elétrica desejada, em dS m${ }^{-1}$, e;

CEai - condutividade elétrica inicial da água de irrigação antes da adição de $\mathrm{NaCl}$, em dS m${ }^{-1}$.

Um condutivímetro digital portátil modelo CD - 880 da fabricante Instrutherm, foi utilizado nas medições da CE atual e desejada durante o preparo das soluções em reservatório de 500,0 L. Este reservatório servia de fonte de captação para o conjunto motor-bomba destinado a conduzir a solução para um sistema de irrigação por gotejamento. O sistema de irrigação foi modificado para se adequar à casualização do delineamento. Para cada nível de CE aplicado, utilizou-se linhas de derivação individuais com válvulas de abertura e fechamento conectadas a tubos de polietileno lisos, os quais foram instalados emissores apenas nas posições dos vasos que receberiam determinado tratamento. Já para o NI variou-se o tempo de aplicação.

Como material vegetal utilizou-se o girassol, cv. Charrua, da Atlântica Sementes LTDA, cultivando-se 10 sementes por unidade experimental. Os tratamentos foram impostos apenas aos 12 dias após semeadura (DAS), quando se contabilizou $80 \%$ de emergência visando obter stand de plantas a serem avaliadas. Aos 22 DAS foi feito desbaste manual, com permanência de uma planta por vaso para proceder com as avaliações fisiológicas.

As avaliações fisiológicas foram realizadas aos 66 DAS, quanto a cultura encontrava-se no estádio reprodutivo R4 (início do florescimento). As plantas foram avaliadas no intervalo entre 7:00 h e 10:00 h da manhã (Maia et al., 2012) em folhas do terço médio e completamente saudáveis, mensurando os parâmetros de trocas gasosas: taxa fotossintética (A, $\mu$ mol $\left.\mathrm{m}^{-2} \mathrm{~s}^{-1}\right)$, taxa transpiratória $\left(\mathrm{E}, \mu \mathrm{mol} \mathrm{m} \mathrm{m}^{-2} \mathrm{~s}^{-1}\right)$, condutância estomática $\left(\mathrm{Gs}, \mathrm{mol} \mathrm{H}_{2} \mathrm{O} \mathrm{m}^{-2} \mathrm{~s}^{-1}\right)$, concentração interna de $\mathrm{CO} 2(\mathrm{Ci}$, $\left.\mu \mathrm{mol} \mathrm{m} \mathrm{s}^{-2}\right)$ e a relação entre a concentração interna e externa de $\mathrm{CO}_{2}(\mathrm{Ci} / \mathrm{Ca})$. Posteriormente determinou-se as razões $\mathrm{A} / \mathrm{Ci}$, A/Gs e A/E. As variáveis citadas foram mensuradas por um medidor portátil de fotossíntese, modelo LCi Light Systems (ADC, BioScientific, Herts, England), acoplado a uma fonte de luz LCI, com densidade de fluxo de fótons igual a $1000 \mu \mathrm{mol} \mathrm{m}^{-2} \mathrm{~s}^{-1}$. A densidade de fluxo de fótons de $1000 \mu \mathrm{mol} \mathrm{m} \mathrm{m}^{-2} \mathrm{~s}^{-1}$ usada no estudo foi aquela utilizada em trabalho anterior realizado por (Maia et al., 2012) com a cultura de girassol e níveis de salinidade.

Avaliou-se também os danos ao aparato fotossintético por meio da fluorescência da clorofila, utilizando um fluorômetro modulado MINI-PAM (Walz, Effeltrich, Germany). Dessa forma, determinou-se o rendimento quântico efetivo do fotossistema II ( $\phi$ PSII), o rendimento quântico potencial do fotossistema II (Fv/Fm) o quenching fotoquímico (qP) e o quenching não-fotoquímico (qNP).

Os dados foram tabulados e as análises estatísticas constaram de análise de variância, que quando significativas, as médias dos fatores qualitativos (CE) foram comparadas por Tukey ( $\mathrm{p}<0,05)$ e as quantitativas (NI) por regressão, determinando-se os modelos que melhor se ajustaram aos dados observados. As análises estatísticas foram realizadas com auxílio do software Sisvar (Ferreira, 2014). Ademais, todos os princípios e rigores metodológicos e científicos estão de acordo com (Pereira et al., 2018; Koche, 2011).

\section{Resultados e Discussão}

Na Tabela 2 consta o resumo da análise de variância onde é possível verificar a interação significativa entre os tratamentos para as variáveis $A, G s, C i, E$ e para as razões $A / C i$ e $A / E$. 
Tabela 2. Resumo da análise de variância para as variáveis de e fluorescência em girassol submetido a níveis de reposição hídrica e condutividades elétricas.

\begin{tabular}{|c|c|c|c|c|c|c|c|c|c|c|c|c|}
\hline \multirow{2}{*}{ FV } & \multirow{2}{*}{ GL } & \multicolumn{11}{|c|}{ Quadrados médios } \\
\hline & & $A$ & Gs & $\mathrm{Ci}$ & $E$ & $\mathrm{Ci} / \mathrm{Ca}$ & $A / C i$ & $A / E$ & $\phi P S I I$ & $F v / F m$ & $q P$ & $q N P$ \\
\hline Bloco & 2 & $2,36^{\mathrm{ns}}$ & $0,027^{\mathrm{ns}}$ & 71,36 & $0,08^{\text {ns }}$ & $0,011^{\mathrm{ns}}$ & $0,00007^{\mathrm{ns}}$ & $0,38^{\mathrm{ns}}$ & $0,009^{\text {ns }}$ & $0,005^{\mathrm{ns}}$ & $0,020^{\mathrm{ns}}$ & $0,025^{\mathrm{ns}}$ \\
\hline NI & 3 & $24,23^{* *}$ & $0,011^{\mathrm{ns}}$ & $631,20^{* *}$ & $1,22^{* *}$ & $0,001^{\mathrm{ns}}$ & $0,00039^{* *}$ & $2,88^{* *}$ & $0,011^{\mathrm{ns}}$ & $0,003^{\mathrm{ns}}$ & $0,015^{\mathrm{ns}}$ & $0,041^{\mathrm{ns}}$ \\
\hline $\mathrm{CE}$ & 1 & $90,32^{* *}$ & $0,002^{\text {ns }}$ & $3772,28^{* *}$ & $0,00^{\mathrm{ns}}$ & $0,004^{\mathrm{ns}}$ & $0,00183^{* *}$ & $4,52^{* *}$ & $0,000^{\mathrm{ns}}$ & $0,000^{\text {ns }}$ & $0,000^{\mathrm{ns}}$ & $0,000^{\mathrm{ns}}$ \\
\hline $\mathrm{NI}^{*} \mathrm{CE}$ & 3 & $27,72^{* *}$ & $0,137^{*}$ & $159,87^{*}$ & $2,36^{* *}$ & $0,000^{\mathrm{ns}}$ & $0,00031^{* *}$ & $1,67^{* *}$ & $0,012^{\text {ns }}$ & $0,007^{\mathrm{ns}}$ & $0,019^{\text {ns }}$ & $0,002^{\mathrm{ns}}$ \\
\hline Resíduo & 14 & 2,41 & 0,274 & 35,53 & 0,17 & 0,002 & 0,000 & 0,20 & 0,008 & 0,005 & 0,014 & 0,027 \\
\hline $\mathrm{CV}(\%)$ & & 7,580 & 24,86 & 2,03 & 7,60 & 6,37 & 7,35 & 11,60 & 21,03 & 12,77 & 20,58 & 22,77 \\
\hline
\end{tabular}

** significativo a 0,01 de probabilidade; ${ }^{*}$ significativo a 0,05 de probabilidade; ${ }^{\text {ns }}$ não significativo pelo teste F; $A$ - Taxa fotossintética; Gs condutância estomática; $\mathrm{Ci}$ - concentração interna de $\mathrm{CO}_{2} ; \mathrm{E}$ - taxa transpiratória,; $\mathrm{Ci} / \mathrm{Ca}$ - razão entre concentração interna e externa de $\mathrm{CO}_{2}$; $A / C i$ - razão entre a taxa fotossintética e a concentração interna de $\mathrm{CO}_{2} ; A / E$ - razão entre a taxa fotossintética e a transpiratória; $\phi P S I I$ rendimento quântico efetivo do fotossistema II; $F v / F m$ - rendimento quântico potencial do fotossistema II; $q P$ - quenching fotoquímico; $q N P$ - quenching não fotoquímico; NI - Nível de irrigação; CE - Condutividade elétrica da água de irrigação; CV - Coeficiente de variação; GL Grau de liberdade; FV - Fonte de variação.

Fonte: Autores (2021).

Para $\mathrm{Ci} / \mathrm{Ca}$ e todos os demais parâmetros de fluorescência $\phi P S I I, F v / F m, q P$ e $q N P$ não houve interações significativas e nem influência isolada dos tratamentos. A fluorescência é capaz de fornecer uma medida útil do desempenho fotossintético dos vegetais. Entretanto, sua principal função é fornecer informações sobre a capacidade de tolerância de uma planta sob estresses ambientais, a medida em que diferentes intensidades danificam o aparelho fotossintético (Maxwell e Johnson, 2000). Dessa forma, analisando isoladamente os parâmetros de fluorescência, percebe-se que os tratamentos não foram suficientes para influencia-los nessa data de avaliação. Contudo, deve-se ter maior atenção para $F v / F m$, uma vez que o resultado indica a manutenção do aparato fotossintético, mais precisamente do fotossistema II (FSII), quando irrigado com água salina. Essa relação $F v / F m$ é um refinado indicador do desempenho fotossintético da planta e valores muito baixos podem indicar estresse e / ou fotoinibição, que consiste em danos a nível de FSII (Maxwell e Johnson, 2000; Araújo et al., 2017) ou, também, regulação negativa da fotossíntese (Jägerbrand \& Kudo, 2016).

Analisando o estresse, Shabala (2017) descreve que não há uma definição concisa e extensa do estresse de plantas que seja suficientemente ampla para acomodar uma gama de "respostas aos estressores", sem incluir todas as alterações fisiológicas em um organismo. Também cita os diferentes conceitos propostos por outros estudiosos, como Taiz \& Zeiger (2012) que o descreve como uma influência desvantajosa exercida na planta por fatores bióticos ou abióticos. Estes conceitos possuem sempre uma visão agronômica, porém, sem consenso e com margem para diferentes interpretações.

As comparações entre as condutividades elétricas para cada nível de irrigação são possíveis serem observadas na Tabela 3, onde está presente o desdobramento de todas as variáveis em que se constatou diferença significativa para interação (tabela 2). 
Tabela 3. Desdobramento da interação entre a condutividade elétrica da água dentro de cada nível de irrigação.

\begin{tabular}{|c|c|c|c|c|c|c|c|c|c|}
\hline \multirow{2}{*}{$\begin{array}{c}\mathrm{CE} \\
\mathrm{dS} \mathrm{m}^{-1}\end{array}$} & \multicolumn{4}{|c|}{ Nível de irrigação (\%) } & \multirow{2}{*}{$\begin{array}{c}\mathrm{CE} \\
\mathrm{dS} \mathrm{m}^{-1}\end{array}$} & \multicolumn{4}{|c|}{ Nível de irrigação (\%) } \\
\hline & 25 & 50 & 75 & 100 & & 25 & 50 & 75 & 100 \\
\hline & \multicolumn{4}{|c|}{$A\left(\mu \mathrm{mol} \mathrm{m} \mathrm{m}^{-2} \mathrm{~s}^{-1}\right)$} & & \multicolumn{4}{|c|}{$E\left(\mu \mathrm{mol} \mathrm{m}{ }^{-2} \mathrm{~s}^{-1}\right)$} \\
\hline 0,6 & $23,08 \mathrm{a}$ & $20,39 \mathrm{a}$ & $16,13 \mathrm{~b}$ & $14,60 \mathrm{~b}$ & 0,6 & $5,61 \mathrm{a}$ & $5,30 \mathrm{~b}$ & $4,99 \mathrm{~b}$ & $5,94 \mathrm{a}$ \\
\hline 3,0 & $22,34 \mathrm{a}$ & $22,76 \mathrm{a}$ & $20,50 \mathrm{a}$ & $24,11 \mathrm{a}$ & 3,0 & $4,05 \mathrm{~b}$ & $6,42 \mathrm{a}$ & $5,86 \mathrm{a}$ & $5,44 \mathrm{a}$ \\
\hline & \multicolumn{4}{|c|}{ Gs $\left(\mathrm{mol} \mathrm{H} \mathrm{H}_{2} \mathrm{O} \mu \mathrm{mol} \mathrm{m}^{-2} \mathrm{~s}^{-1}\right)$} & & \multicolumn{4}{|c|}{$\mathrm{A} / \mathrm{Ci}$} \\
\hline 0,6 & $0,83 \mathrm{a}$ & $0,62 \mathrm{a}$ & $0,57 \mathrm{a}$ & $0,68 \mathrm{a}$ & 0,6 & $0,08 \mathrm{a}$ & $0,07 \mathrm{~b}$ & $0,05 \mathrm{~b}$ & $0,05 \mathrm{~b}$ \\
\hline 3,0 & $0,42 \mathrm{~b}$ & $0,78 \mathrm{a}$ & $0,84 \mathrm{a}$ & $0,59 \mathrm{a}$ & 3,0 & $0,08 \mathrm{a}$ & $0,08 \mathrm{a}$ & $0,07 \mathrm{a}$ & $0,09 \mathrm{a}$ \\
\hline & \multicolumn{4}{|c|}{$C i\left(\mu \mathrm{mol} \mathrm{m}^{-2} \mathrm{~s}^{-1}\right)$} & & \multicolumn{4}{|c|}{$A / E$} \\
\hline 0,6 & $302,61 \mathrm{a}$ & 291,99 a & 326,07 a & 305,27 a & 0,6 & $4,11 \mathrm{~b}$ & $3,84 \mathrm{a}$ & $3,25 \mathrm{a}$ & $2,46 b$ \\
\hline 3,0 & $278,32 \mathrm{~b}$ & $281,16 \mathrm{~b}$ & $292,19 \mathrm{~b}$ & $273,97 \mathrm{~b}$ & 3,0 & $5,62 \mathrm{a}$ & $3,55 \mathrm{a}$ & $3,52 \mathrm{a}$ & $4,43 \mathrm{a}$ \\
\hline
\end{tabular}

A - Taxa fotossintética, Gs - condutância estomática, $\mathrm{Ci}$ - concentração interna de $\mathrm{CO}_{2}, \mathrm{E}$ - taxa transpiratória, $\mathrm{A} / \mathrm{Ci}$ - razão entre a taxa fotossintética e a concentração interna de $\mathrm{CO}_{2}, \mathrm{~A} / \mathrm{E}$ - razão entre a taxa fotossintética e a taxa transpiratória e CE - Condutividade elétrica da água de irrigação. Obs - Médias seguidas pela mesma letra, na coluna, não diferem significativamente entre si pelo teste de Tukey a 0,05 de probabilidade.

Fonte: Autores (2021).

A condutância estomática foi limitada pela aplicação de água salina $\left(3,0 \mathrm{dS} \mathrm{m}^{-1}\right)$ apenas no NI de $25 \%$, demonstrando que o sal aplicado via irrigação não influenciou na regulação dos estômatos do girassol cv. Charrua quando se tem níveis de água no solo superiores a $25 \%$ da evapotranspiração de referência. Entretanto, as menores concentrações internas de $\mathrm{CO}_{2}(\mathrm{Ci})$ observadas no girassol quando irrigado com água salina, não estão relacionadas com a Gs, mas sim pelas altas taxas fotossintéticas encontradas nessas plantas.

Em oposição as plantas irrigadas com água sem adição de sal $\left(0,6 \mathrm{dS} \mathrm{m}^{-1}\right)$ a $G s$ obteve sempre valores iguais ou superiores ao das plantas irrigadas com água salina, entretanto a fotossíntese não obteve o mesmo comportamento, sendo inferior nos maiores NI (75\% e 100\%), o que explica justamente as maiores $\mathrm{Ci}$, já que o $\mathrm{CO}_{2}$ que se difundiu sem nenhuma restrição para o interior da folha não foi fixado pelo processo fotossintético na mesma proporção observada nas plantas irrigadas com água salina, provavelmente devido a uma diminuição na atividade de enzimas envolvidas no processo de fixação de $\mathrm{CO}_{2}$ (Borba et al., 2017).

Considerando $A$ ou também chamada de fotossíntese líquida, percebe-se que a água salina não limitou a fotossíntese do girassol, pelo contrário. Nos níveis mais elevados de irrigação (75 e 100\%) o uso de água salina favoreceu a fotossíntese do girassol, com incremento de $21,3 \%$ na $A$ para o nível de irrigação com $75 \%$ da transpiração de referência e $39,4 \%$ de incremento no nível de $100 \%$ (tabela 3). A razão A/Ci é um importante indicador que reflete a eficiência instantânea de carboxilação, em que retrata a fotossíntese líquida da planta a partir da concentração interna de $\mathrm{CO}_{2}$. Em outras palavras, quando não ocorre uma limitação difusiva por via estomática, o aumento de $\mathrm{A} / \mathrm{C} i$ significa aumento da eficiência fotossintética da planta e consequente aumento na produção de fotoassimilados.

A eficiência instantânea de carboxilação obteve comportamento semelhante com $A$, com maiores eficiências quando irrigado com água salina, prioritariamente nos maiores níveis de água (tabela 3). Este comportamento pode ser explicado devido essas plantas que vivem em ambientes com estresse salino responderem diferentemente ao potencial hídrico do solo do que aquelas que crescem sob condições não salinas (Shoukat, et al., 2018), através de importantes mecanismos de defesa presente em algumas espécies ou materiais genéticos melhorados, sendo um deles o ajuste osmótico, através do acúmulo de solutos que podem ajudar a alcançar o equilíbrio osmótico, a captação de água para manter o crescimento (Flowers e Colmer, 
2008) e alteração do módulo de elasticidade da parede celular, pois plantas com tecidos mais elásticos mantêm maiores quantidades de água na saturação total e, portanto, no ponto de perda do turgor, elas podem ter maior capacitância hidráulica em relação ao volume da célula (Lambers et al., 2008; Bartlett et al., 2012).

Ao estudar o comportamento do girassol irrigado com água salina, Gomes et al. (2015) observaram uma redução de 33,92\% na fotossíntese líquida, 33,94\% na transpiração e 41,26\% na condutância estomática, considerando a aplicação de água de irrigação com CE equivalente a 0,8 e 3,0 $\mathrm{dSm}^{-1}$. Em estudo realizado por Torabian et al. (2016), foram observados decréscimos na ordem de 25,77\% em A, 26,83\% na Gs e 6,85\% para $F v / F m$, parâmetros estes avaliados em girassol cultivado sob 0 e $100 \mathrm{mM}$ de $\mathrm{NaCl}$. É válido enfatizar que todos esses resultados mostram redução de processos fisiológicos relacionados a à fotossíntese, quando ocorre o aumento dos níveis de $\mathrm{CE}$ em água de irrigação.

Portanto o resultado deste estudo é estimulante, no que tange a escolha de cultivares resistentes ao estresse salino. Entretanto é importante salientar que o aumento da eficiência fotossintética nem sempre resulta em aumento de produtividade, uma vez que, processos diversos como a translocação de fotoassimilados para órgãos de interesse, competem com investimentos em proteção e/ou manutenção, os quais são dispendiosos em qualquer cultura, principalmente sujeita a estresses.

A transpiração obteve comportamento diferente da fotossíntese líquida, onde se observou semelhança apenas no NI75\% (tabela 3), sendo contrário a Kozai (2016) ao descrever que, o aumento da fotossíntese é precedido do aumento da captação de $\mathrm{CO}_{2}$ e, inevitavelmente, da elevação da taxa transpiratória. Para a $A / E$, que de acordo com Feldman et al. (2017) corresponde a eficiência no uso da água instantânea $\left(E U A_{i}\right)$, os valores foram superiores nas condições de água salina dentro dos NI de 25 e 100\%, sendo condizente com o resultado obtido por Melo et al. (2016) que ao estudarem a cultura do pimentão constataram maiores eficiências no uso da água com incrementos na concentração de sal na água de irrigação.

Como já exposto anteriormente a CE de 3,0 dS m ${ }^{-1}$ não limitou os estômatos do girassol e para essa mesma CE observa-se que o NI estimado responsável pela maior Gs foi de 68,33\% (Figura 1) dentro de um comportamento quadrático, enquanto que a maior taxa transpiratória para essa CE foi observada no NI de 70,27\% (Figura 2) com o mesmo comportamento, o que é esperado, pois a transferência de vapor de água da folha para o ar atmosférico ocorre predominantemente por via estomática (Defraeye et al., 2014; Mengis et al., 2015). Portanto o ambiente que proporcionar maiores Gs também será o responsável para maior $E$ devido à alta correlação positiva entre esses parâmetros.

O menor NI foi responsável pela maior $G s$ dentro da CE sem adição de sal, demonstrando que o déficit aplicado não foi suficiente para limitar a abertura estomática, que se constitui em um dos primeiros processos fisiológicos a ser desencadeado pela seca em plantas (Figura 1), a fim de diminuir a perda de água para o ambiente e otimizar seu uso em condições de seca (Makbul et al., 2011). Já na irrigação salina houve limitação da condutância estomática no nível mínimo e máximo de irrigação (Figura 1), evidenciando que para se alcançar um alta $G s$ foi necessário manter um nível de água equilibrado entre 50 e $75 \%$. 
Figura 1. Condutância estomática de girassol em função do nível de irrigação com condutividade elétrica de $0,6 \mathrm{dS} \mathrm{m} \mathrm{m}^{-1}$ (linha pontilhada) e 3,0 dS m $\mathrm{m}^{-1}$ (linha contínua).

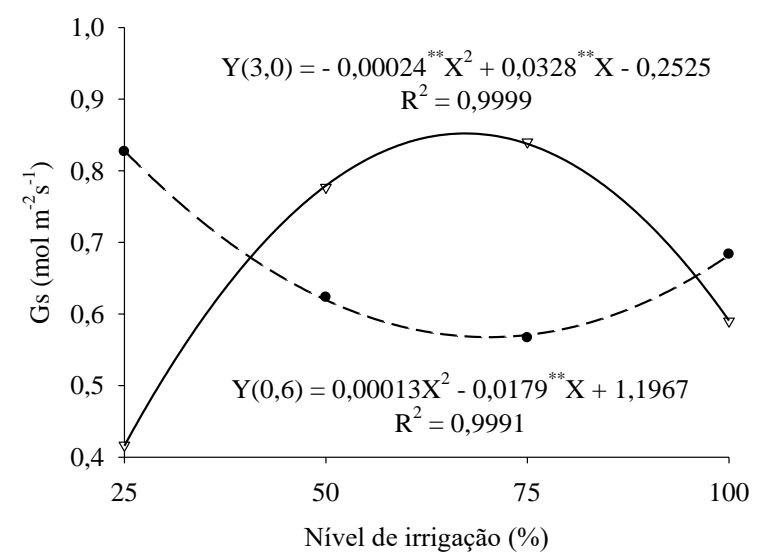

Figura 2. Transpiração do girassol em função do nível de irrigação com condutividade elétrica de $0,6 \mathrm{dS} \mathrm{m} \mathrm{m}^{-1}$ (linha pontilhada) e 3,0 $\mathrm{dS} \mathrm{m}^{-1}$ (linha contínua).

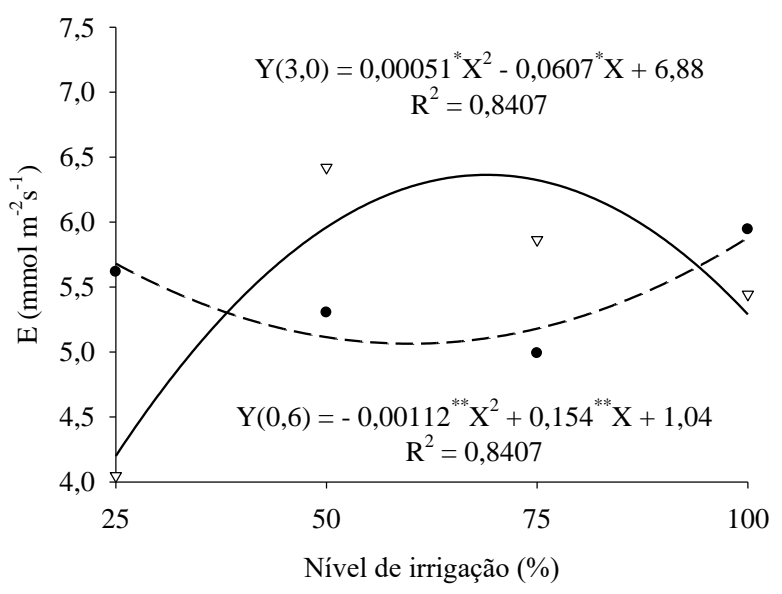

Fonte: Autores.

Houve um decréscimo na taxa fotossintética com aumento dos NI dentro da CE de 0,6 $\mathrm{dS} \mathrm{m}^{-1}$ (Figura 3), de modo que o menor NI foi o responsável pela maior taxa fotossintética, sendo um forte evidencia para a resistência da cultivar a ambientes com baixas disponibilidades hídricas. Acrescenta-se que a manutenção da taxa fotossintética, mesmo com baixos teores de água no solo é o que dita a resistência à seca de uma planta (Mota \& Cano, 2016). A Ci dentro da irrigação com água salina obteve o mesmo comportamento que Gs e E, com maiores valores encontrados no NI de 62,17\% (Figura 4), demonstrando que a $G$ s favoreceu a entrada de $\mathrm{CO}_{2}$ na câmara subestomática, posteriormente destinada ao processo fotossintético.

Figura 3. Taxa fotossintética do girassol em função do nível de irrigação com condutividade elétrica de $0,6 \mathrm{dS} \mathrm{m}^{-1}$

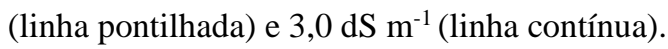

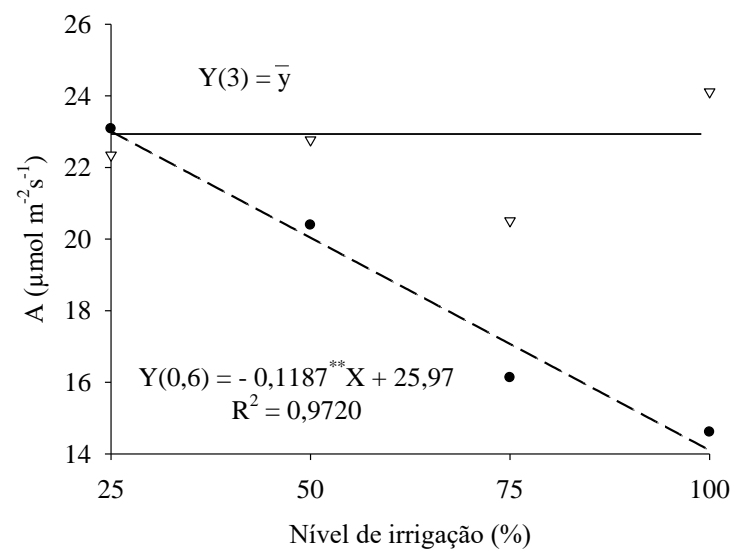

Figura 4. Concentração interna de $\mathrm{CO}_{2}$ do girassol em função do nível de irrigação com condutividade elétrica de 3,0 dS m $\mathrm{d}^{-1}$ (linha pontilhada) e 3,0 dS m $\mathrm{m}^{-1}$ (linha contínua).

\footnotetext{
Fonte: Autores.
}

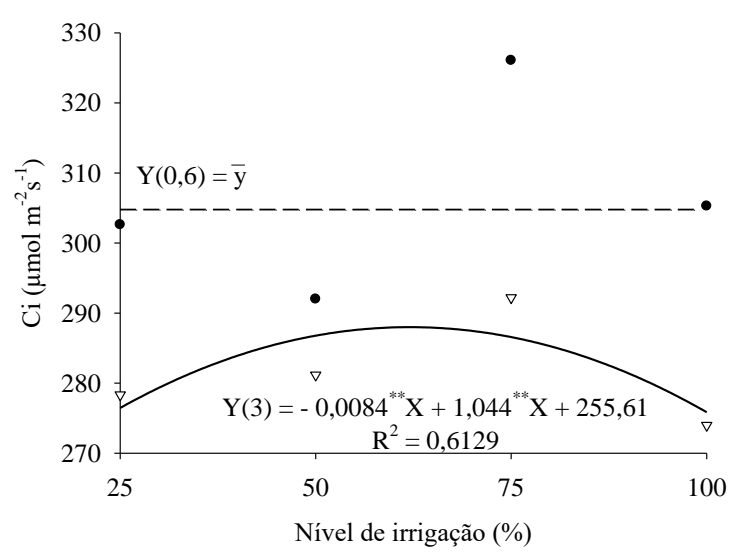


A razão $\mathrm{A} / \mathrm{Ci}$ dentro da $\mathrm{CE}$ de $0,6 \mathrm{dS} \mathrm{m}^{-1}$ reforça a hipótese de resistência a baixos teores de água no solo presente na cv estudada, pois, à medida que houve diminuição do NI a eficiência instantânea de carboxilação aumentou, indicando ainda que a irrigação com mais de $25 \%$ da transpiração de referência foi prejudicial para esses parâmetros fisiológicos (Figura 5), além de ter apresentado o mesmo comportamento para a $A / E$ (Figura 6).

Figura 5. Razão $\mathrm{A} / \mathrm{Ci}$ do girassol em função do nível de irrigação com condutividade elétrica de $0,6 \mathrm{dS} \mathrm{m} \mathrm{m}^{-1}$ (linha pontilhada) e $3,0 \mathrm{dS} \mathrm{m}^{-1}$ (linha contínua).

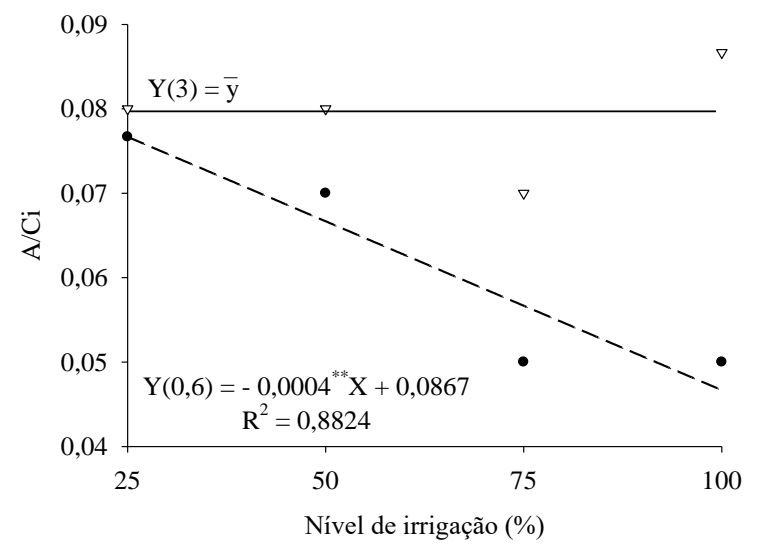

Figura 6. Razão A/E do girassol em função do nível de irrigação com condutividade elétrica de $0,6 \mathrm{dS} \mathrm{m} \mathrm{m}^{-1}$ (linha pontilhada) e 3,0 $\mathrm{dS} \mathrm{m}^{-1}$ (linha contínua).

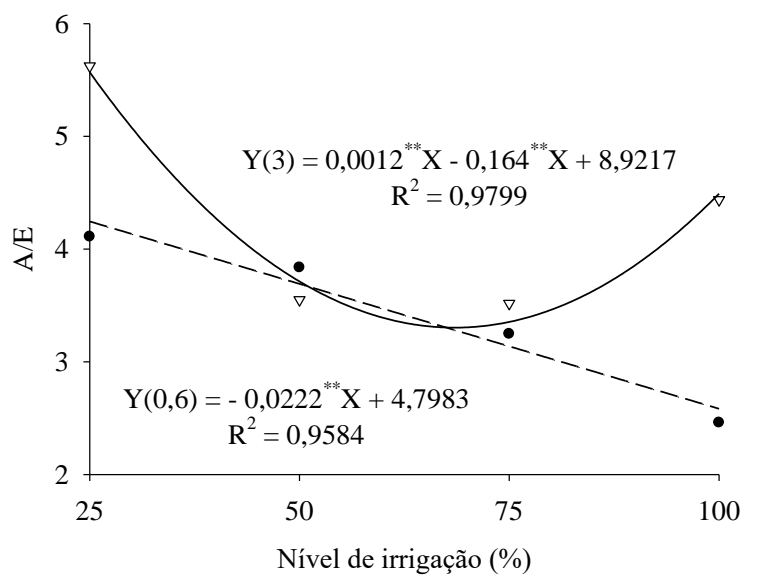

Fonte: Autores.

As menores $A / E$ dentro da $\mathrm{CE}$ de 3,0 dS m m $^{-1}$ foram observadas entre os NI de 50 e $75 \%$ (Figura 6), tendo um comportamento inverso das variáveis $E$ e $G s$, pois com aumento dessas variáveis é comum que essas eficiências de uso da água instantâneas reduzam, devido a $G s$ e $E$ serem os processos que regulam a perda de água da planta para o ambiente. Na irrigação sem água salina a $A / E$ foi decrescente com o aumento dos NI (Figura 6), não acompanhando o padrão encontrado na irrigação com água salina, apesar deste comportamento, isso enaltece a capacidade em que a planta possui em controlar a perda de água para o ambiente mesmo com disponibilidade hídrica menor no solo, sendo uma característica muito importante para cultivo em ambientes em que a água é uma grande limitação.

Considerando os resultados encontrados nesse estudo, em relação as concentrações de sal na água e os níveis de irrigação é possível inferir que a cv. Charrua é um potencial material de cultivo em ambientes irrigados com água de CE alterada, visto que, o mesmo não demonstrou reduções nos parâmetros fisiológicos avaliados e em algumas variáveis de interesse foi possível notar até mesmo respostas positivas quanto adição de sal na água; bem como ser um material interessante para ambientes com baixa disponibilidade hídrica, por apresentar incremento na eficiência fotossintética com a redução do nível de água no solo. Selecionar cultivares resistentes a essas limitações é muito importante, pois dentre os estresses abióticos, a salinidade tornou-se um grande problema, pois restringe a produtividade em quase $50 \%$ da terra irrigada globalmente (Ruan et al. 2010).

\section{Conclusão}

Os processos fisiológicos do girassol cultivar Charrua como condutância estomática, fotossíntese líquida, eficiência instantânea de carboxilação e no uso da água não foram limitados pela irrigação salina, sendo que em alguns atributos houve 
até incrementos; dentro da irrigação sem sal os menores níveis de irrigação foram responsáveis pelos maiores valores desses mesmos atributos.

Os resultados indicam que a cultivar utilizada é um potencial material vegetal resistente tanto a baixos níveis de água no solo quanto ao uso de água salina na irrigação.

São necessários mais estudos aplicando mais intervalos de condutividade elétrica da água de irrigação, com o intuito de definir limites fisiológicos de tolerância da cultivar Charrua.

\section{Agradecimentos}

Os autores agradecem ao Ministério da Ciência, Tecnologia, Inovações e Comunicações (MCTIC), à Fundação de Amparo à Pesquisa do Estado de Goiás (FAPEG), ao Conselho Nacional de Desenvolvimento Científico e Tecnológico (CNPq) e ao Instituto Federal Goiano (IFGoiano) pelo auxílio financeiro ao presente projeto de pesquisa.

\section{Referências}

Araújo, R. P., Almeida, A. A. F., Pereira, L. S., Mangabeira, P. A. O., Souza, J. O., Pirovani, C. P., Ahnert, D., \& Baligar, V. C. (2017). Photosynthetic, antioxidative, molecular and ultrastructural responses of young cacao plants to cd toxicity in the soil. Ecotoxicology and Environmental Safety, $144,148-157$. https://doi.org/10.1016/j.ecoenv.2017.06.006

Ashraf, M., Shahzad, S. M., Akhtar, N., Imtiaz, M., \& Ali, A. (2017). Salinization/sodification of soil and physiological dynamics of sunflower irrigated with saline-sodic water amending by potassium and farm yard manure. Journal of Water Reuse and Destination, 7(4), 476-487. https://doi.org/10.2166/wrd.2016.053

Bartlett M.K., Scoffoni C., \& Sack L. (2012). The determinants of leaf turgor loss point and prediction of drought tolerance of species and biomes: a global meta-analysis. Ecology Letters, 15, 393-405. https://doi:10.1111/j.1461-0248.2012.01751.x

Borba, M. E. A., Maciel, G. M., Júnior, E. F., Júnior, C. M., Marquez, G. R., Silva, I. G., \& Almeida, R. S. (2017). Gas exchanges and water use efficiency in the selection of tomato genotypes tolerant to water stress. Genetics and molecular research: GMR, 16(2), 1-9. http://dx.doi.org/10.4238/gmr16029685.

Dalchiavon, F. C., Malacarne, B. J., \& Carvalho, C. G. P. (2016). Características agronômicas de genótipos de girassol (Helianthus annuus L.) em segunda safra no Chapadão do Parecis - MT. Revista de Ciências Agrárias, 39(1), 178-186. http://dx.doi.org/10.19084/RCA15049

Defraeye, T., Derome, D., Verboven, P., Carmeliet, J., \& Nicolai, B. (2014). Cross-scale modelling of transpiration from stomata via the leaf boundary layer. Annals of botany, 114(4),711-723. https://doi.org/10.1093/aob/mct313

Dutra, C. C., Prado, E. A. F., Paim, L. R., \& Scalon, S. P. Q. (2018). Desenvolvimento de plantas de girassol sob diferentes condições de fornecimento de água Semina: Ciências Agrária, 33(6), 2657-2667. http://www.uel.br/revistas/uel/index.php/semagrarias/article/view/8200

Feldman, A. B., Leung, H., Baraoidan, M., Elmido-Mabilangan, A., Canicosa, I., Quick, W. P., Sheehy, J., \& Murchie, E. H. (2017). Increasing leaf vein density via mutagenesis in rice results in an enhanced rate of photosynthesis, smaller cell sizes and can reduce interveinal mesophyll cell number. Frontiers in Plant Science, 8, 1-10. https://doi.org/10.3389/fpls.2017.01883

Feng, G., Zhang, Z., Wan, C., Lu, P., \& Bakour, A. (2017). Effects of saline water irrigation on soil salinity and yield of summer maize (Zea mays L.) in subsurface drainage system. Agricultural Water Management, 193, 205-213. https://doi.org/10.1016/j.agwat.2017.07.026

Ferreira, D. F. Sisvar: a guide for its bootstrap procedures in multiple comparisons. (2014). Ciência e Agrotecnologia, 38(2), 109-112. http://dx.doi.org/10.1590/S1413-70542014000200001

Flowers T. J, \& Colmer T. D. 2008. Salinity tolerance in halophytes. New Phytologist, 179, 945-963. http://dx.doi:10.1111/j.1469-8137.2008.02531.x

Gomes, K. R., Sousa, G. G., Lima, F. A., Viana, T. V. A., Azevedo, B. M., \& Silva, G. L. (2015). Irrigação com água salina na cultura do girassol (Helianthus annuиs L.) em solo com biofertilizante bovino. Irriga, 20(4), 680-693. http://dx.doi.org/10.15809/irriga.2015v20n4p680

Guedes Filho, D. H., Santos, J. B., Gheyi, H. R., Cavalcante, L. F., \& Santos Júnior, J. A. (2015). Componentes de produção e rendimento do girassol sob irrigação com águas salinas e adubação nitrogenada. Irriga, 20(3), 514-527. http://dx.doi.org/10.15809/irriga.2015v20n3p514

Habibi, G. (2017). Physiological, photochemical and ionic responses of sunflower seedlings to exogenous selenium supply under salt stress. Acta Physiologiae Plantarum, 39(213), 1-9. https://doi.org/10.1007/s11738-017-2517-3

Jägerbrand, A. K., \& Kudo, G. (2016). Short-term responses in maximum quantum yield of PSII (Fv/Fm) to ex situ temperature treatment of populations of bryophytes originating from different sites in Hokkaido, northern Japan. Plants, 5(22), 1-7. https://doi.org/10.3390/plants5020022

Koche, J. C. (2011). Fundamentos de metodologia científica. Petrópolis: Vozes. http://www.adm.ufrpe.br/sites/ww4.deinfo.ufrpe.br/files/F undamentos_de_Metodologia_Cienti\%CC\%81fica.pdf

Kozai, T. (2016). Why LED lighting for urban agriculture? LED lighting for urban agriculture. Springer Singapore. 
Research, Society and Development, v. 10, n. 12, e97101220199, 2021

(CC BY 4.0) | ISSN 2525-3409 | DOI: http://dx.doi.org/10.33448/rsd-v10i12.20199

Lambers H, Chapin F. S., \& Pons T. L. (2008). Plant water relations. Plant physiological ecology. Springer New York.

Lira, R. M., Santos, A. N., Silva, J. S., Barnabé, J. M. C., Barros, M. S., \& Soares, H. R. (2015). A utilização de águas de qualidade inferior na agricultura irrigada. Revista Geama - Environmental Science, 1(3), 841-862. Disponível em: http://www.journals.ufrpe.br/index.php/geama/article/view/514/1438

Lustri, E. A., Silva, B. T., Peruchi, D. R. E., Moura, I. A., \& Fluminhan, A. (2017). Avaliação do desempenho agronômico de cultivares de girassol (Helianthus annuus L.) no cultivo em safrinha na região Oeste Paulista. Forúm Ambiental da Alta Paulista, 13(1), 37-51. http://dx.doi.org/10.17271/1980082713120171488

Maia, F. M. A., Costa, A. C., Castro, J. N., \& Megguer, C. A. (2012). Trocas gasosas em plantas de girassol submetidas à sainidade. In: V Congresso Brasileiro de Mamona, II Simpósio Internacional de Oleaginosas Energéticas \& I Fórum Capixaba de Pinhão Manso, 2012, Guarapari. Desafios e Oportunidades: Anais... Campina Grande: Embrapa Algodão, 196.

Makbul, S., Saruhan-Guler, N., Durmus, N, \& Guven, S. (2011). Changes in Anatomical and Physiological Parameters of Soybean Under Drought Stress. Turk. J. Bot. 35(4), 369-377. http://dx.doi.org/10.3906/bot-1002-7

Maxwell, K., \& Johnson, G. N. (2000). Chlorophyll fluorescence - a practical guide. Journal of Experimental Botany, 51(345), 659-668. https://doi.org/10.1093/jexbot/51.345.659

Melo, H. F. D., Souza, E. R. D., Duarte, H. H., Cunha, J. C., \& Santos, H. R. (2017). Gas exchange and photosynthetic pigments in bell pepper irrigated with saline water. Revista Brasileira de Engenharia Agrícola e Ambiental, 21(1), 38-43. http://dx.doi.org/10.1590/1807-1929/agriambi.v21n1p38-43

Mengis, N., Keller, D. P., Eby, M., \& Oschlies, A. (2015). Uncertainty in the response of transpiration to $\mathrm{CO}_{2}$ and implications for climate change. Environmental Research Letters, 10(9), 1-9. http://dx.doi.org/10.1088/1748-9326/10/9/094001

Mila, A. J., Ali, M. H., Akanda, A. R., Rashid, M. H. O., \& Rahman, M. A. (2017). Effects of deficit irrigation on yield, water productivity and economic return of sunflower. Congent Food \& Agriculture, 3, 1-14. https://doi.org/10.1080/23311932.2017.1287619

Miorini, T. J. J., Saad, J. C. C., \& Menegale, M. L. (2011). Supressão de água em diferentes fases fenológicas do feijoeiro (Phaseolus vulgaris L.). Irriga, 16(4), 360-368. http://dx.doi.org/10.15809/irriga.2011v16n4p360

Mota, C. S., \& Cano, M. A. O. (2016). Respostas fisiológicas de plantas jovens de macaúba a condições de seca cíclica. Pesquisa Florestal Brasileira, 36(87), 225-234. https://doi.org/10.4336/2016.pfb.36.87.1061

Patane, C., Cosentino, S. L., \& Anastasi, U. (2017). Sowing time and irrigation scheduling effects on seed yield and fatty acids profile of sunflower in semiarid climate. International Journal of Plant Production, 11(1), 17-32. https://dx.doi.org/10.22069/ijpp.2017.3307

Pereira A. S. et al. (2018). Metodologia da pesquisa científica. UFSM. https://repositorio.ufsm.br/bitstream/handle/1/15824/Lic_Computacao_MetodologiaPesquisa-Cientifica.pdf?sequence $=1$

Richards, L. A. (1954). Diagnosis and improvement of saline and alkali soils. Agricultural Handbook 60. Washington D.C.: U.S. Salinity Laboratory, 1954, 160p. https://www.ars.usda.gov/ARSUserFiles/20360500/hb60_pdf/hb60complete.pdf

Ruan C. J, da Silva J. A. T, Mopper S, Qin P, \& Lutts S. (2010). Halophyte improvement for a salinized world. Critical Reviews in Plant Sciences, 29, 329359. http://dx.doi:10.1080/07352689.2010.524517

Santos Júnior, J. A., Gheyi, H. R., Cavalcante, A. R., Dias, N. S., \& Medeiros, S. S. (2018). Produção e pós-colheita de flores de girassóis sob estresse salino em hidroponia de baixo custo. Engenharia Agrícola, 36, 3, 420-432. http://www.scielo.br/pdf/eagri/v36n3/1809-4430-eagri-36-3-0420.pdf

Santos, M. R., \& Brito, C. F. B. Irrigação com água salina, opção agrícola consciente. Revista Agrotecnologia, 7(1), 33-41. http://www.revista.ueg.br/index.php/agrotecnologia/article/download/5175/pdf

Shabala, S. (2017). Plant stress physiology. Centre for Agriculture and Biosciences International.

Shoukat, E., Aziz, I., Ahmed, M. Z., Abideen, Z., \& Khan, M. A. (2018). Growth patterns of Phragmites karka under saline conditions depend on the bulk elastic modulus. Crop and Pasture Science, 69(5), 535-545. https://doi.org/10.1071/CP17195

Taiz, L., \& Zeiger, E. (2012). Fisiologia vegetal. (5a ed.), Artmed.

Torabian, S., Zahedi, M., \& Khoshgoftar, A. H. (2016). Effects of foliar spray of two kinds of zinc oxide on the growth and ion concentration of sunflower cultivars under salt stress. Journal of plant nutrition, 39(2), 172-180. https://doi.org/10.1080/01904167.2015.1009107 